\title{
EDITORIAL
}

\section{The Intra-aortic Balloon Pump (IABP)- An Essential Component of Modern Cardiac Care}

In modern cardiology practice, intra-aortic balloon pump (IABP) has become an invaluable component in some critical patient care. The Intra-aortic balloon pump (IABP) is a mechanical device that increases myocardial oxygen perfusion and at the same time increasing cardiac output. A specialised arterial catheter, which has a helium filled balloon is inserted percutaneously into the descending aorta approximately 1 inch from the left subclavian artery. This catheter is attached to the IABP which pumps helium into the balloon during ventricular diastole thereby increasing myocardial perfusion, draws back the helium prior to ventricular systole thus decreasing ventricular afterload. Increasing cardiac output increases coronary blood flow and therefore myocardial oxygen delivery.These actions combine to decrease myocardial oxygen demand and increase myocardial oxygen supply. A computer-controlled mechanism inflates the balloon with helium from a cylinder during diastole, usually linked to either an electrocardiogram (ECG) or a pressure transducer at the distal tip of the catheter. Helium is used because its low viscosity allows it to travel quickly through the long connecting tubes, and has a lower risk of causing an embolism should the balloon rupture.

The IABP device was pioneered at Grace Sinai Hospital in Detroit during the early 1960s by Dr. Adrian Kantrowitz and his team. The device was developed for use in heart surgery by Dr. David Bregman in 1976 at NewYorkPresbyterian Hospital in New York City. The first clinical implant was performed at Maimonides Medical Center, Brooklyn, N.Y. in Oct., 1967. The patient, a 48 year old woman, was in cardiogenic shock and unresponsive to traditional therapy. An IABP was inserted by a cut down on the left femoral artery. Pumping was performed for approximately 6 hours. Shock reversed and the patient was discharged. The size of the original balloon was 15 French but eventually 9 and 8 French balloons were developed. A second operation removed the balloon. Since 1979 the placement of the balloon has been modified using the Seldinger technique.

IABP increases myocardial and systemic blood flow in the following incidences: LV failure, unstable refactory angina, septic shock, mechanical complications secondary to AMI, bridge to cardiac transplantation, valvular disease, prophylaxis prior to cardiac surgery, post cardiac surgery cardiogenic shock and provides support for failed angioplasty and valvuloplasty.

There are some contraindications: Severe aortic insufficiency - as the balloon inflates, blood may be forced across the valve thereby overloading the ventricle and increasing cardiac work, Aortic dissection and Aortic aneurysm - the increased pressure generated by counterpulsation may cause the aneurysm to rupture, Severe peripheral vascular disease may limit the ability to advance the catheter through atherosclerotic vessels, Severe coagulopathy and Prosthetic Aortofemoral grafts are also contraindications for IABP.

Since the device is placed in the femoral artery and aorta it could provoke ischemia, and compartment syndrome. The leg is at highest risk of becoming ischemic if femoral artery it is supplied becomes obstructed. Placing the balloon too distally may induce occlusion of the renal artery and subsequent renal failure. Other possible complications are cerebral embolism during insertion, infection, dissection of the aorta or iliac artery, perforation of the artery and hemorrhage in the mediastinum. Mechanical failure of the balloon itself is also a risk which entails vascular surgery to remove under that circumstance. After balloon removal there is also a risk of 'embolic shower' from micro clots that have formed on the surface of the balloon, and can lead to peripheral thrombosis, myocardial ischemia, hemodynamic decompensation, and late pseudoaneurysm. Obviously complications are there, but considering cost benefit ratio IABP has become an essential equipment in modern cardiology practice. It should be available in all modern cardiac centres where cathlab facilities exist.
Dr. Mohammad Safiuddin
Associate Professor of Cardiology
Bangabandhu Sheikh Mujib Medical University, Dhaka
E-mail: safiuddin1960@yahoo.com 